Signal \& Image Processing : An International Journal (SIPIJ) Vol.6, No.3, June 2015

\title{
FUZZY CLUSTERING BASED GLAUCOMA DETECTION USING THE CDR
}

\author{
Thresiamma Devasia ${ }^{1}$, Poulose Jacob ${ }^{2}$ and Tessamma Thomas ${ }^{3}$ \\ ${ }^{1}$ Department of Computer Science, \\ Assumption College Changanacherry, Kerala, India \\ ${ }^{2}$ Department of Computer Science, \\ Cochin University of Science and Technology, Kerala, India \\ ${ }^{3}$ Department of Electronics, Cochin University of Science and Technology, Kerala, India
}

\begin{abstract}
Glaucoma is a serious eye disease, overtime it will result in gradual blindness. Early detection of the disease will help prevent against developing a more serious condition. A vertical cup-to-disc ratio which is the ratio of the vertical diameter of the optic cup to that of the optic disc, of the fundus eye image is an important clinical indicator for glaucoma diagnosis. This paper presents an automated method for the extraction of optic disc and optic cup using Fuzzy C Means clustering technique combined with thresholding. Using the extracted optic disc and optic cup the vertical cup-to-disc ratio was calculated. The validity of this new method has been tested on 365 colour fundus images from two different publicly available databases DRION, DIARATDBO and images from an ophthalmologist. The result of the method seems to be promising and useful for clinical work.
\end{abstract}

\section{KEYWORDS}

fundus image, optic disc, optic cup, Cup-to-Disc Ratio , Fuzzy C Means Clustering

\section{INTRODUCTION}

Glaucoma is a disease of increased pressure within the eyeball. The disease is mostly caused due to increased intraocular pressure (IOP) resulting from a malfunction or malformation of the eye's drainage structures. If left untreated, it would lead to degeneration of optic nerve and retinal fibers. Early diagnosis of glaucoma through analysis of the neuro-retinal optic disc (OD) and optic cup (OC) area is crucial. The increase in pressure results in immoderate amount of stress to be put to the attachment of the optic nerve to the eye. Lack of treatment for glaucoma can lead to permanent blindness. Early detection of the disease will help prevent against developing a more serious condition. The fundus images are used for diagnosis by trained clinicians to check for any abnormalities or any change in the retina. Important anatomical structures captured in a fundus image are blood vessels, OD, OC, and macula for a normal retina. An image of a diseased retina may also contain many visible symptoms of the eye-disease. In a healthy retinal image the OD usually appears as a circular shaped bright yellowish object which is partly covered with vessels. 
Signal \& Image Processing : An International Journal (SIPIJ) Vol.6, No.3, June 2015

The OC is the cupping of the optic nerve and that means the size of the depression in the middle of the nerve when viewed from the front of the eye. When there is damage to the optic nerve, the cupping increases. Changes in the OD and OC can indicate the presence, current state and progression of glaucoma [1][2].

Since the colour fundus images provide early signs of certain diseases such as diabetes, glaucoma etc., colour fundus images are used to track the eye diseases by the ophthalmologists. Figure 1 shows the important features of a retinal colour fundus image.

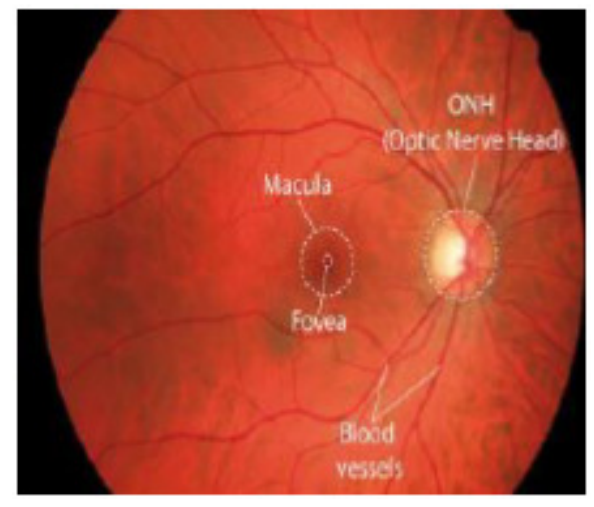

Figure1. Colour Fundus Image

Diseases with symptoms on the fundus images are very complex. Several main symptoms of diseases expressed on the disk optic, blood vessels, haemorrhage and lesion areas on the retinal background. For the OD, differences in the color, shape, edge or vasculature may signify a pathological change or may just be part of the wide spectrum of normality. The physiological cup varies in shape, size and depth, and any enlargement of the cup is one parameter used in the assessment of glaucoma. Figure 2 shows the colour fundus image of a normal eye and glaucomatous eye.

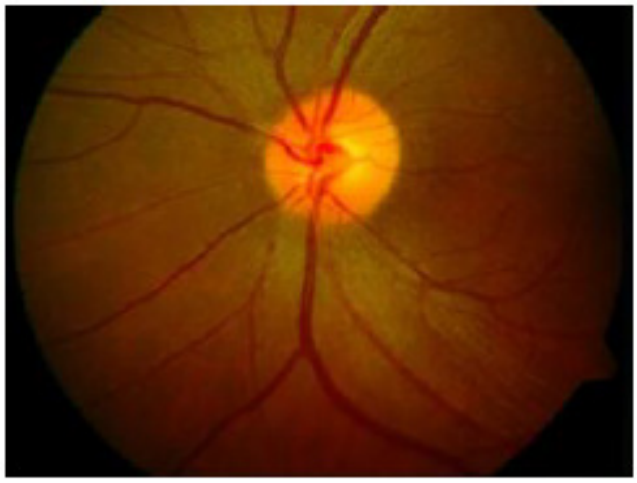

(i)

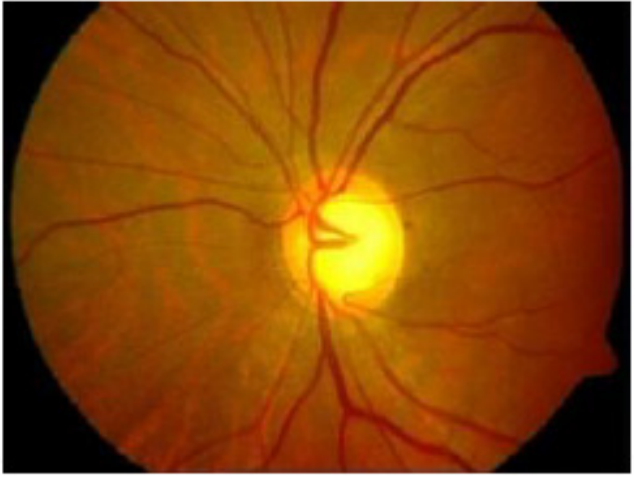

(ii)

Figure 2. (i) Fundus image of a normal eye (ii) Glaucomatous eye fundus image 
Signal \& Image Processing : An International Journal (SIPIJ) Vol.6, No.3, June 2015

One of the hallmarks of glaucoma is the optic nerve damage, which is characterized by cupping of the optic nerve. Even a normal optic nerve has a small amount of cupping. However, the patients with glaucoma tend to have larger cupping than normal subjects. The cup-to-disc ratio (CDR) of normal subjects is typically around 0.2 to 0.4 as it is shown in Figure 2(i). However, with glaucoma, there is progressive loss of optic nerve fibers, and consequent increase in the cup size of the optic nerve[3]. Figure 2(ii) shows a glaucomatous fundus image. Nowadays, CDR is manually determined by trained ophthalmologists and has to be diagnosed by using retinal images from special cameras which are expensive. These are limitations of potential in mass screening for early detection. This paper focused on developing a new method that can provide suitable way to compute CDR result which give the most accurate result and prevent from current limitation.

This paper is organized as follows:

Section 2 presents a brief survey of existing literature. Section 3 describes the materials used for the present work. A new algorithm to efficiently extract OD and OC in ocular fundus images and computation of CDR are given in Section 4. The results are presented in Section 5, and Conclusions are given in the final Section 6.

\section{LITERATURE SURVEY}

Different techniques are described in the literature for OD and OC extraction for the computation of the CDR. The Active Shape Model (ASM) based optical disk detection is implemented by Huiqi et al.[4]. The initialization of the parameters for this model is based on Principal Component Analysis technique. The faster convergence rate and the robustness of the technique are proved by experimental results. Huajun Ying et al. [5] designed a fractal-based automatic localization and segmentation of optic disc in retinal images. K. Shekar [6] developed a method for OD segmentation using Hussain, A.R. et al. [7] proposed a method for optic nerve head segmentation using genetic active contours. J.Liu et al. proposed a technique to extract optic disc and cup in order to determine cup to disc ratio. Optic disc is extracted using a variational level set method and the detected contour is uneven due to influence of blood vessels. Detection of cup boundary was performed using intensity and threshold level set approach. Thresholding techniques produced better results for both high and low risk retinal images. An ellipse fitting is used to smoothen the boundary $[8,9]$.

Zhuo Zhang et al. [10] designed a convex hull based neuro-retinal optic cup ellipse optimization technique. Wong, D.W.K. et al. [11] developed SVM-based model optic cup detection for glaucoma detection using the cup to disc ratio in retinal fundus images. Joshi G.D. et al. [12] developed vessel bend-based cup segmentation in retinal images. Shijian Lu et al. [13] proposed a background elimination method for the automatic detection of OD. Gopal Datt Joshi developed a deformable model guided by regional statistics to detect the OD boundary. Cup boundary detection scheme is based on Lab color space and the expected cup symmetry. This method uses sector wise information and give rise to fewer false positives and hence better specificity. Error value computed is less for a normal image than for a glaucomatous image [14]. Morphological operations were used for locating the optic disc in retinal images by Angel Suero et al. [15].

In this paper, a new algorithm based on Fuzzy C-Means Clustering (FCM) technique combined with thresholding, is used for OD and OC extraction. This new method, firstly, extracts the OD 
Signal \& Image Processing : An International Journal (SIPIJ) Vol.6, No.3, June 2015

and $\mathrm{OC}$ of the colour fundus image and computes the vertical CDR automatically. This is an efficient method for the automatic screening of colour fundus image for CDR computation.

\section{MATERIALS AND MethodS}

The fundus images used in these experiments are taken from publicly available databases DRION and DIARATDB0 and, images from Giridhar Eye Institute, Kochi, Kerala. The CDRs obtained from an ophthalmologist is used as ground truth for the evaluation.

\section{DEVELOPED ALGORITHM}

The new approach is composed of four steps. The channels of the colour retinal are separated. The blood vessels are removed, applying the contrast adjustment to enhance the low contrast image image. The Fuzzy C Means combined with thresholding is applied on the red channel of the input image for the extraction of the OD and the same technique is applied on the green channel of the input image for the extraction of OC. The CDR is computed using the ratio of vertical diameter of $\mathrm{OC}$ and $\mathrm{OD}$.

\subsection{Preprocessing}

The preprocessing step excludes variations due to image acquisition, such as inhomogeneous illumination. In preprocessing, techniques such as morphological operations and contrast enhancement are applied on the input image. The following sections include different preprocessing operations used in this paper.

\subsubsection{Preprocessing steps for Optic Disc Extraction}

\subsubsection{Selection of Red Channel}

From the previous studies it is shown that even though the green component of an RGB retinography is the one with highest contrast, the OD is often present in the red field as a welldefined white shape, brighter than the surrounding area [16]. Therefore the red channel of the $\mathrm{RGB}$ colour images is used for the extraction of OD regions in the retinal fundus images.

\subsubsection{Removal of Blood Vessels}

Since blood vessels within the OD are strong distracters, they should be erased from the image beforehand. In this method a morphological closing operation is performed on the red channel. The dilation operation first removes the blood vessels and then the erosion operation approximately restores the boundaries to their former position.

Closing : $C(A, B)=A \bullet B=E(D(A,-B),-B)$

where $\mathrm{A}$ is the red channel of the input image and B is a 10x10 symmetrical disc structuring element, to remove the blood vessels[17,18]. C is the resultant vessel free, smoothed output image. 
Signal \& Image Processing : An International Journal (SIPIJ) Vol.6, No.3, June 2015

\subsubsection{Preprocessing steps for Optic Cup Extraction}

\subsubsection{Selection of Green Channel}

The green channel has low contrast variation which gives more differentiation between the blood vessel and OC. The green channel, therefore, is selected for the extraction of the OC of the retinal image.

\subsubsection{Removal of Blood Vessels}

Blood vessels in the green channel were removed using a morphological closing procedure,

$$
I 2(I, B)=A \bullet B=E(D(I,-B),-B)
$$

where $\mathrm{I}$ is the green channel of the input image and $\mathrm{B}$ is an $8 \mathrm{x} 8$ symmetrical disc structuring element, to remove the blood vessels[17,18]. I2 is the smoothed, vessel free output image. Figure 3 shows the preprocessing operations on the input image.

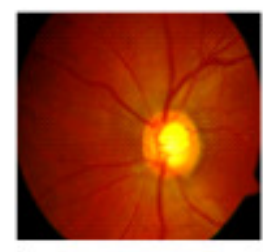

(a)

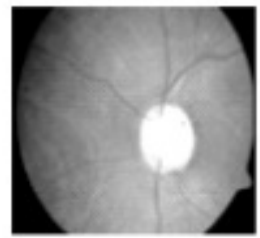

(b)

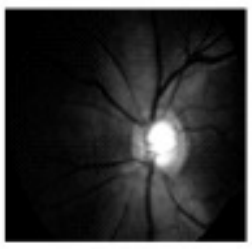

(d)

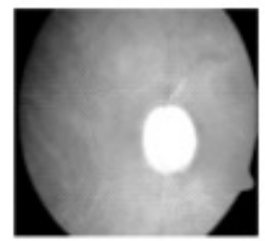

(c)

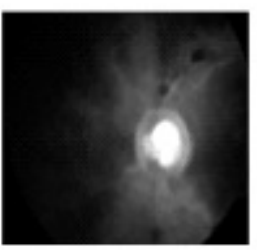

(e)

Figure 3.The preprocessing steps (a) Input Image (b) Red channel (c ) Blood vessel free image (d) Green channel (e) Blood vessel free image

\subsection{Feature Extraction}

Medical image segmentation is a difficult task due to the complexity of segmentation. Because of its simplicity and efficiency, threshold segmentation is wildly used in many fields. Assessment of $\mathrm{OD}$ and $\mathrm{OC}$ is important in discriminating between normal and pathological retinal images. The OD is a bright pattern of the fundus image. Recently, many studies on the use of fundus images in extracting OD and OC have been reported. FCM clustering with thresholding is used in this work for the extraction of OD and OC. 
Signal \& Image Processing : An International Journal (SIPIJ) Vol.6, No.3, June 2015

\subsection{Fuzzy C Means Clustering with Thresholding}

The new method is a combination of fuzzy algorithm, C Means clustering and thresholding. Clustering involves the task of dividing data points into homogeneous classes or clusters so that items in the same class are as similar as possible and items in different classes are as dissimilar as possible. Clustering can also be thought of as a form of data compression, where a large number of samples are converted into a small number of representative prototypes or clusters. Different types of similarity measures may be used to identify classes depending on the data and the application, where the similarity measure controls the formation of the clusters. In the following new method intensity value is used as the similarity measure. Thresholding is one of the most powerful techniques for image segmentation, in which the pixels are partitioned depending on their intensity value.

\subsubsection{Fuzzy C-Means Clustering Algorithm}

FCM clustering is a clustering technique and it employs fuzzy partitioning such that a data point can belong to all groups with different membership grades between 0 and 1 . It is an iterative algorithm. The aim of FCM is to find cluster centers (centroids) that minimize a dissimilarity function. Corresponding to each cluster center, this algorithm works by assigning membership to each data point on the basis of the difference between the cluster center and the data point. The more the data is near to the cluster center, the more is its membership towards the particular cluster center. It is obvious that the summation of membership of each data point should be equal to one.

$$
\begin{aligned}
& \sum_{i=1}^{c} u_{i j}=1, \forall j=1, \ldots, n \\
& \mathrm{~d}_{\mathrm{k}}=\left[\sum_{\mathrm{j}=1}^{\mathrm{m}_{\mathrm{j}}}\left[\mathrm{x}_{\mathrm{kj}}-\mathrm{v}_{\mathrm{j}}\right]^{2}\right]^{1 / 2},
\end{aligned}
$$

where $\mathrm{X}_{\mathrm{kj}}$ is data element, $\mathrm{d}_{\mathrm{ik}}$ is the distance matrix and $\mathrm{V}_{\mathrm{ij}}$ is the element of the cluster center vector.

The dissimilarity function which is used in FCM is given Equation (5)

$$
J\left(U, c_{1}, c_{2}, \ldots, c_{c}\right)=\sum_{i=1}^{c} J_{i}=\sum_{i=1}^{c} \sum_{j=1}^{n} u_{i j}{ }^{m} d_{i j}{ }^{2}
$$

$\mathrm{u}_{\mathrm{ij}}$ is between 0 and 1 ;

$c_{i}$ is the centroid of cluster $i$;

$\mathrm{d}_{\mathrm{ij}}$ is the Euclidian distance between $\mathrm{i}^{\text {th }}$ centroid (ci) and $\mathrm{j}^{\text {th }}$ data point;

$\mathrm{m} \epsilon[1, \infty]$ is a weighting exponent.

To reach a minimum of dissimilarity function there are two conditions. These are given in Equation (6) and Equation (7) 
Signal \& Image Processing : An International Journal (SIPIJ) Vol.6, No.3, June 2015

$$
\begin{gathered}
c_{i}=\frac{\sum_{j=1}^{n} u_{i j}{ }^{m} x_{j}}{\sum_{j=1}^{n} u_{i j}^{m}} \\
u_{i j}=\frac{1}{\sum_{k=1}^{c}\left(\frac{d_{i j}}{d_{k j}}\right)^{2 /(m-1)}}
\end{gathered}
$$

This algorithm determines the following steps [4].

Step1. Randomly initialize the membership matrix (U) that has constraints in Equation 7.

Step2. Calculate centroids $\left(\mathrm{C}_{\mathrm{i}}\right)$ by using Equation (6).

Step3. Compute dissimilarity between centroids and data points using equation (5). Stop if its improvement over previous iteration is below a threshold.

Step4. Compute a new U using Equation (7). Go to Step 2 [19][20].

By iteratively updating the cluster centers and the membership grades for each data point, FCM iteratively moves the cluster centers to the apt location within a data set. To accommodate the introduction of fuzzy partitioning, the membership matrix (U) is randomly initialized according to Equation (7).

The Fuzzy Logic Toolbox command line function fcm is used for generating clusters, and in this paper three clusters are generated from the vessel free enhanced image. The fcm function iteratively moves the cluster centers to the right location within the data set. The outputs are 3 cluster centers $\mathrm{C} 1, \mathrm{C} 2$ and $\mathrm{C} 3$ and membership function matrix $\mathrm{M}$ with membership-grades, which is the intensity value of each pixel.

\subsection{Thresholding}

Thresholding is the operation of converting a multilevel image into a binary image i.e., it assigns the value of 0 (background) or 1 (objects or foreground) to each pixel of an image based on a comparison with some threshold value $\mathrm{T}$ (intensity or colour value) [17][18]. By applying the threshold $\mathrm{T}$ on an image, the image is converted to a binary image. The following formula (8) [13] is used for the binary image extraction.

$$
\mathrm{I}_{\mathrm{T}}(\mathrm{x}, \mathrm{y})=\left\{\begin{array}{l}
1, \text { if } \mathrm{I}(\mathrm{x}, \mathrm{y})>\mathrm{T} \\
0, \text { if } \mathrm{I}(\mathrm{x}, \mathrm{y})<=\mathrm{T},
\end{array}\right.
$$

where $\mathrm{I}$ is the input image, $\mathrm{T}$ is the threshold and $\mathrm{I}_{\mathrm{T}}$ is the binary image after thresholding. 
Signal \& Image Processing : An International Journal (SIPIJ) Vol.6, No.3, June 2015

\subsection{Extraction of Optic Disc}

The main feature of the OD is that it is having the highest intensity. Therefore the highest intensity is used as the threshold for the OD extraction. The threshold $\mathrm{T}$ is computed using the following method. From the generated clusters, first the cluster with maximum membership grade is found, and the corresponding grades are assigned with the same identification label. From the smoothed image, pixels with this gray level value are accessed, the average of the maximum and minimum intensity values are computed to obtain the threshold value $T_{1}$.

$$
\text { i.e., } T_{1}=\frac{1}{2}[\operatorname{Max}(\text { data }(\text { value }))+\operatorname{Min}(\text { data }(\text { value }))]
$$

In the above equation, data represents the data points of the smoothed red channel image and label represents the cluster value with the highest membership grade. By applying the threshold $T_{1}$ on the smoothed image $I_{S}$ the image is converted to a binary image. The formula (9) is used for the binary image extraction.

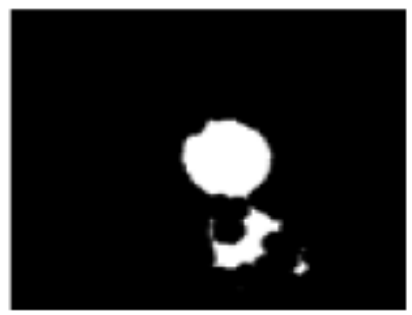

Figure 4. The extracted binary image

Since the OD is of circular shape, the OD region selection process needs to be made specific to the circular region. So the largest connected component $R_{i}$ whose shape is approximately circular is selected using the compactness measure

$$
C(\mathrm{Ri})=\frac{\mathrm{P}(\mathrm{Ri})}{4 \pi \mathrm{A}(\mathrm{Ri})}
$$

where, $\mathrm{P}\left(\mathrm{R}_{\mathrm{i}}\right)$ is the perimeter of the region $\mathrm{R}_{\mathrm{i}}$ and $\mathrm{A}\left(\mathrm{R}_{\mathrm{i}}\right)$ is the area of the region $\mathrm{R}_{\mathrm{i}}$. The binary image with the compactness smaller than the pre-specified value, ( 5 in the present study) is considered as the optic disc approximation. Thus using the condition $\mathrm{C}<5$, extraction of round objects is done, eliminating those objects that do not meet the criteria. In some cases the extracted image contains small unwanted objects. The erosion operation is used to remove these objects [21].

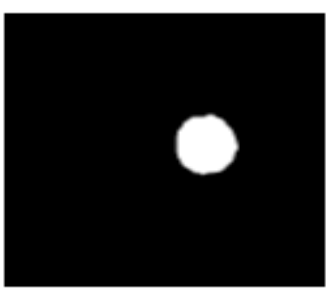

Figure 5 Extracted Optic Disc 
Signal \& Image Processing : An International Journal (SIPIJ) Vol.6, No.3, June 2015

The mean of the rows and columns form the centroid (Y1, X1) of the OD.

$$
\begin{aligned}
& \mathrm{Y} 1=\frac{1}{\mathrm{~m}} \sum_{\mathrm{i}=1}^{\mathrm{m}} \text { row } 1 \mathrm{i} \\
& \mathrm{X} 1=\frac{1}{\mathrm{n}} \sum_{\mathrm{i}=1}^{\mathrm{n}} \operatorname{col} 1 \mathrm{i},
\end{aligned}
$$

where $\mathrm{m}$ is the number of rows and $\mathrm{n}$ is the number of columns.

From the above coordinates of the optic disc the minimum coordinates (ymin1, xmin1) is calculated. The distance between the centroid and (ymin1, xmin1) represents the radius of the disc.

$$
\mathrm{R}_{\mathrm{OD}}=\mathrm{Y} 1-\mathrm{ymin} 1
$$

where $\mathrm{R}_{\mathrm{OD}}$ is the radius of the optic disc.

\subsection{Extraction of Optic Cup}

The above mentioned FCM clustering with thresholding is applied on the smoothed green channel for the extraction of OC.

The following algorithm includes four steps [4].

Step1. Randomly initialize the membership matrix (U) that has constraints in Equation (6).

Step2. Calculate centroids $\left(\mathrm{C}_{\mathrm{i}}\right)$ by using Equation (7).

Step3. Compute dissimilarity between centroids and data points using equation (5). Stop if its improvement over previous iteration is below a threshold.

Step4. Compute a new U using Equation (6). Go to Step 2[19][20].

The threshold values $T_{2}$ is calculated using the following equation.

$$
\mathrm{T}_{2}=\frac{1}{2}[\operatorname{Max}(\text { data }(\text { value }))+\operatorname{Min}(\text { data (value) })],
$$

where data represents the data points of the vessel free green channel and label represents the cluster value with the highest membership grade.

Since the OC is the brightest portion in the green channel, thresholding with threshold $\mathrm{T}_{2}$ in im2 bw function helps to extract OC. This function returns the binary image forming the object OC.

The average of the rows and columns forms the centroid $(\mathrm{Y} 2, \mathrm{X} 2)$ of the OC.

$$
\mathrm{Y} 2=\frac{1}{\mathrm{~m} 2} \sum_{\mathrm{i}=1}^{\mathrm{m} 2} \text { row } 2 \mathrm{i}
$$


Signal \& Image Processing : An International Journal (SIPIJ) Vol.6, No.3, June 2015

$$
\mathrm{X} 2=\frac{1}{\mathrm{n} 2} \sum_{\mathrm{i}=1}^{\mathrm{n} 2} \operatorname{col} 2 \mathrm{i},
$$

where $\mathrm{m} 2$ is the number of rows and $\mathrm{n} 2$ is the number of columns.

From the above coordinates of the optic cup the minimum coordinates (ymin2, xmin2) is calculated. The Euclidian distance between the centroid and (ymin1, xmin1) returns the radius of the cup.

$$
\mathrm{R}_{\mathrm{OC}}=\mathrm{Y} 2-\mathrm{ymin} 2,
$$

where $\mathrm{R}_{\mathrm{OC}}$ is the radius of the cup.

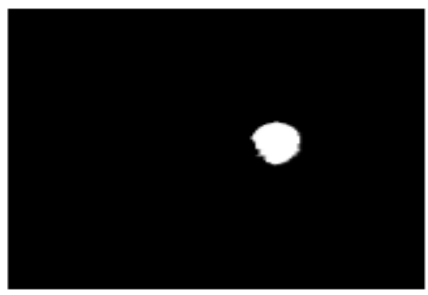

Figure 6. Extracted Optic cup

The method can be used to extract the OD and OC from right eye and left eye images. Figure 7 and Figure 8 show the results of extractions.

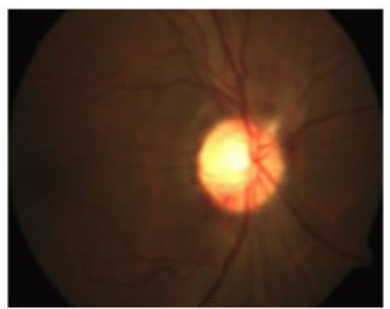

(i)

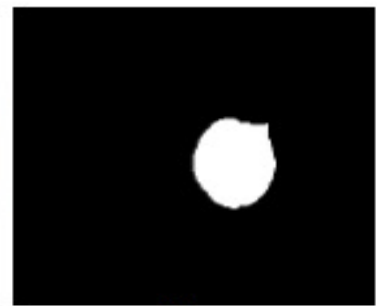

(ii)

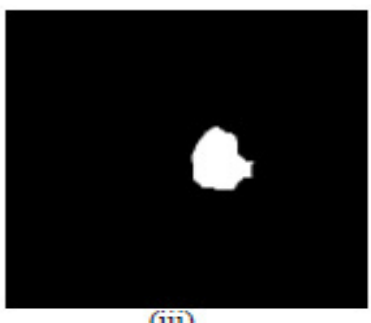

(iii)

Figure 7 (i) Input image of a right eye (ii) Extracted optic disc (iii) Extracted optic cup

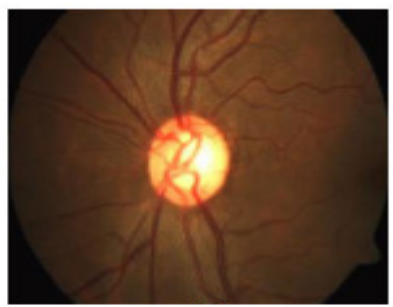

(i)

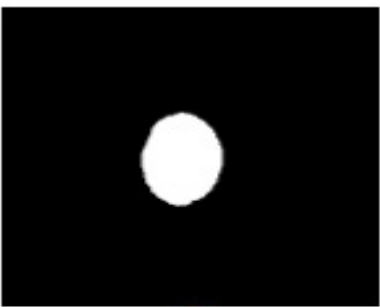

(ii)

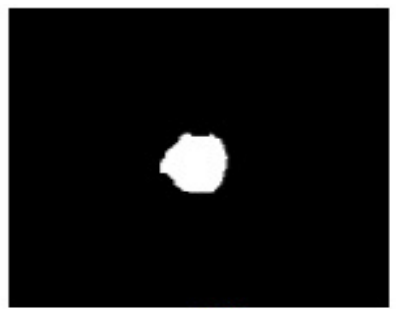

(iii)

Figure 8 (i) Input image of a left eye (ii) Extracted optic disc (iii) Extracted optic cup 
Signal \& Image Processing : An International Journal (SIPIJ) Vol.6, No.3, June 2015

\subsection{Computation of CDR}

The manual method uses the ratio of the vertical diameter of OC and OD for the computation of CDR. From the segmented OD the minimum row coordinate ymin1 and maximum row coordinate $\mathrm{y}_{\max I}$ are calculated. The Euclidian distance between these coordinates is the vertical diameter of the OD, ODvdiam .

ODvdiam $=y \max 1-y \min 1$

Similarly from the segmented $\mathrm{OC}$ the minimum row coordinate $\mathrm{y}_{\min 2}$ and maximum row coordinate $\mathrm{y}_{\max 2}$ are calculated. The Euclidian distance between these coordinates is the vertical diameter of the OC, OCvdiam.

OCvdiam $=y \max 2-y \min 2$

The CDR is calculated using the following formula

$$
\mathrm{CDR}=\mathrm{OC} v \text { diam } / \mathrm{OD} v \text { diam }
$$

The following figure shows the OD vertical diameter ODvdiam and OC vertical diameter OCvdiam of the input image.

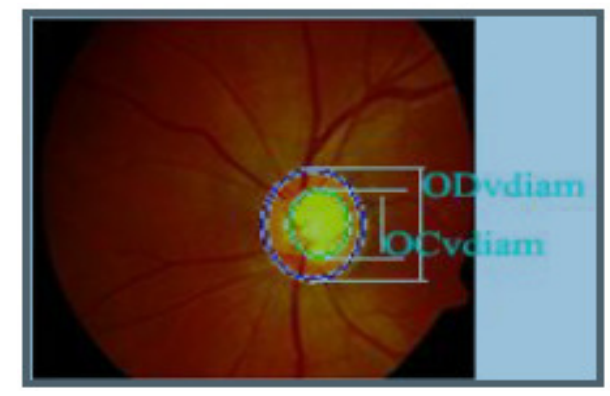

Figure 9 Optic Disc vertical diameter ODvdiam and Optic Cup vertical diameter OCvdiam

\subsection{Glaucoma Detection}

If the CDR is 0.4 or less refers to a relatively healthy looking optic nerve. If the CDR is greater than .4 is suspicious of glaucoma. While there is no one CDR that separates normal from glaucoma, the CDR greater than 0.6 or 0.7 is glaucomatous and often requires further testing to rule out glaucoma. CDR $>.7$ is considered as advanced stage glaucoma. As glaucoma progresses, the CDR enlarges (as more optic nerve fiber dies off), and the patient may start to develop peripheral vision loss. 
Signal \& Image Processing : An International Journal (SIPIJ) Vol.6, No.3, June 2015

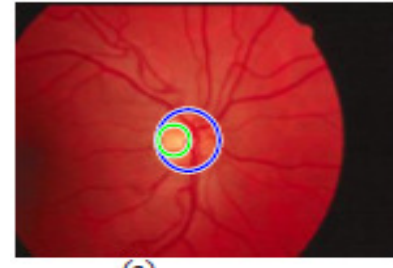

(a) $=0.2328$

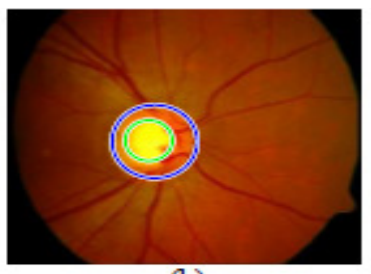

$\mathrm{CDR}=\stackrel{(b)}{51555}$

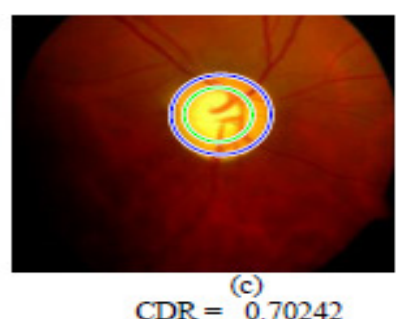

$C D R=0.70242$

Figure 10 (a) Normal (b) Early Stage Glaucoma (c) Advanced Stage Glaucoma

\section{RESULTS AND DISCUSSION}

The automatic computation of CDR is required for automatic detection of glaucoma using retinal fundus images. This study thus brings to light simple but efficient methods for the extraction of $\mathrm{OD}$ and $\mathrm{OC}$ in retinal images. The CDR values are also automatically calculated. The new method is evaluated on the basis of the ground truth data, where, vertical CDR values are obtained from an expert ophthalmologist. The method was applied on 365 retinal color fundus images. The performance evaluation is done by making use of the comparison of obtained CDR with the clinical CDR.

\subsection{Image Data Sets}

\subsubsection{DRION Database}

It has 110 retinal images with each image having the resolution of $600 \times 400$ pixels and the optic disc annotated by two experts with 36 landmarks. The mean age of the patients was 53.0 years (standard Deviation 13.05), with $46.2 \%$ male and 53.8\% female and all of them were Caucasian ethnicity $23.1 \%$ patients had chronic simple glaucoma and $76.9 \%$ eye hypertension. The images were acquired with a colour analogical fundus camera, approximately centered on the $\mathrm{ONH}$ and they were stored in slide format. In order to have the images in digital format, they were digitized using a HP-PhotoSmart-S20 high-resolution scanner, RGB format, resolution 600x400 and 8 bits/pixel.

\subsubsection{The DIARETDB0 Database}

The DIARETDB0 Database images were captured using an FOV of $50^{\circ}$ and the size of each image is $1500 \times 1152 \times 3$. Out of the 130 images of the DIARETDB0 database, 20 have normal architecture and 110 have various types of pathology.

\subsubsection{Images from the ophthalmologist}

125 images from Giridhar Eye Institute, Kochi was also used in this paper. All the images were obtained using Carlzeiss fundus camera. In total 5 are normal images and remaining 120 are diseased and the size of each image is $576 \times 768 \times 3$.

\subsection{Implementation}

The new algorithm was applied on 365 images obtained from the above mentioned databases and ophthalmologists. 
Signal \& Image Processing : An International Journal (SIPIJ) Vol.6, No.3, June 2015

\subsection{Performance Evaluation}

The performance evaluation is done using the following parameters.

\subsubsection{Accuracy}

The accuracy of the technique was evaluated quantitatively by comparing the obtained vertical CDR values with ophthalmologists' ground-truth vertical CDR values. Fifteen examples of detailed results of performance measurement using FCM clustering combined with thresholding are displayed in Table I using fifteen test images of DRION database and fifteen test images from the ophthalmologist.

Table I CDR Comparison Table shows the comparison of clinical CDR values with CDR values obtained using the new method.

\begin{tabular}{|l|l|l|l|l|l|l|}
\hline Images & $\begin{array}{c}\text { Clinical } \\
\text { CDR } \\
(\mathbf{1})\end{array}$ & $\begin{array}{l}\text { Obtained } \\
\text { CDR } \\
(2)\end{array}$ & $\begin{array}{l}\text { Difference } \\
(\mathbf{1})-(\mathbf{2})\end{array}$ & $\begin{array}{l}\text { Clinical } \\
\text { CDR of } \\
\text { DRION } \\
\text { Database } \\
(\mathbf{3})\end{array}$ & $\begin{array}{l}\text { Obtained } \\
\text { CDR }\end{array}$ & $\begin{array}{l}\text { (4) } \\
\text { Difference } \\
\text { (3)-(4) }\end{array}$ \\
\hline Image 1 & 0.5000 & 0.6082 & 0.1082 & 0.3333 & 0.4000 & 0.0667 \\
\hline Image 2 & 0.5714 & 0.6231 & 0.0517 & 0.5734 & 0.5310 & 0.0424 \\
\hline Image 3 & 0.6666 & 0.5505 & 0.1161 & 0.6666 & 0.6080 & 0.0586 \\
\hline Image 4 & 0.8517 & 0.7871 & 0.0646 & 0.7000 & 0.7543 & 0.0543 \\
\hline Image 5 & 0.7142 & 0.6412 & 0.073 & 0.6578 & 0.5816 & 0.0762 \\
\hline Image 6 & 0.4864 & 0.4173 & 0.0691 & 0.5625 & 0.5045 & 0.0580 \\
\hline Image 7 & 0.7060 & 0.6275 & 0.0785 & 0.4062 & 0.4995 & 0.0933 \\
\hline Image 8 & 0.9000 & 0.8367 & 0.0633 & 0.5000 & 0.6020 & 0.1020 \\
\hline Image 9 & 0.6801 & 0.7287 & 0.0486 & 0.6097 & 0.6696 & 0.0626 \\
\hline Image 10 & 0.9026 & 0.8206 & 0.0820 & 0.6410 & 0.6610 & 0.0200 \\
\hline Image 11 & 0.4631 & 0.4012 & 0.0619 & 0.5162 & 0.4011 & 0.1151 \\
\hline Image 12 & 0.6267 & 0.5151 & 0.1116 & 0.5010 & 0.5362 & 0.0352 \\
\hline Image 13 & 0.5147 & 0.4736 & 0.0411 & 0.6097 & 0.5215 & 0.0882 \\
\hline Image 14 & 0.7318 & 0.7180 & 0.0138 & 0.7408 & 0.6943 & 0.0465 \\
\hline Image 15 & 0.4265 & 0.3162 & 0.1103 & 0.6981 & 0.6208 & 0.0773 \\
\hline Mean Difference & & 0.07292 & Mean Difference & 0.06643 \\
\hline
\end{tabular}

\subsubsection{Detection of Glaucoma}

Based on the CDR values the images are classified into three categories such as normal, early stage glaucoma and advanced stage glaucoma. The method is applied on 110 images from DRION database, 130 images from Diaretdb0 and 125 images from an ophthalmologist. Figure 11 shows the detection of different stages of glaucoma based on CDR. 
Signal \& Image Processing : An International Journal (SIPIJ) Vol.6, No.3, June 2015

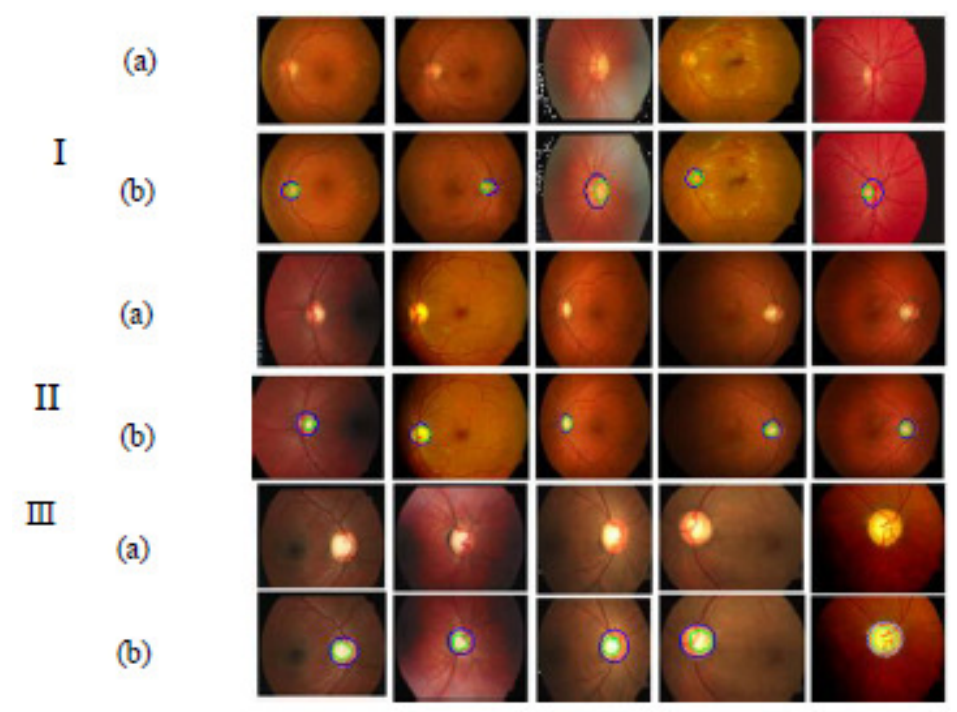

Figure11. (a) Input Image (b) Segmented image I- Normal II - Early Stage

Glacoma III- Advanced Stage Glaucoma

\section{CONCLUSION}

This paper presents a new fuzzy based approach for glaucoma detection. The results presented in this paper show that the new methodology offers a reliable and robust solution for glaucoma detection. This automated method is very useful for the automatic screening of retinal images. However the present method has the following limitations. It is assumed that the OD and OC are brighter than the surrounding pixels and therefore cannot handle retinal images with a relatively dark OD. Hence advanced extraction methods are required for future studies and research.

\section{REFERENCES}

[1] Juan Xu, Hiroshi Ishikawa, Gadi Wollstein, Richard A. Bilonick, Kyung R. Sung,Larry Kagemann, Kelly A. Townsend, and Joel S. Schuman, "Automated Assessment of the Optic Nerve Head on Stereo Disc Photographs", Invest Ophthalmol Vis Sci. Jun 2008; 49(6), pp. 2512-2517, 2008.

[2] Thitiporn Chanwimaluang and Guoliang Fan, "An efficient algorithm for extraction of anatomical structures in retinal images", Proc. of International Conference on Image Processing, Vol. 1, pp. 1093-1096,2003

[3] Chisako Muramatsu, Toshiaki Nakagawab, Akira Sawadac, Yuji Hatanakad, Takeshi Haraa, Tetsuya Yamamotoc, Hiroshi Fujitaa "Determination of cup and disc ratio of optical nerve head for diagnosis of glaucoma on stereo retinal fundus image pairs", Medical Imaging 2009: Computer-Aided Diagnosis, Proc. of SPIE, Vol. 7260, pp.72603L-1 - 72603L-8, 2009

[4] Huiqi Li, Opas Chutatape "Boundary detection of optic disc by a modified ASM method", The Journal of the Pattern Recognition Society, Vol. 36, pp. 2093-2104,2003

[5] Huajun Ying, Ming Zhang and Jyh-Charn Liu, "Fractal-Based Automatic Localization and Segmentation of Optic Disc in Retinal Images", 28th Annual International Conference of the IEEE Engineering In Medicine and Biology Society (EMBS), 2007

[6] S. Sekhar, "Automated Localization Of Optic Disk And Images", Proc. 16th European Signal Processing Conference (EUSIPCO 2008), Lausanne, Switzerland, August 25-29, 2008.

[7] Hussain, A.R., "Optic nerve head segmentation using genetic active Contours", International Conference on Computer and Communication Engineering, 2008,ICCCE 2008, 13-15 May 2008, pp.783 - 787, Univ. Teknikal Malaysia, Melaka, May 2008. 
Signal \& Image Processing : An International Journal (SIPIJ) Vol.6, No.3, June 2015

[8] J.Liu,J.H.Lim, and H.Li, "ARGALI":An automatic cup to disc ratio measurement system for Glaucoma analysis using Level set Image processing," in SPIE Medical Imaging, San Diego, USA, Feb 2008.

[9] J. Liu, D. W .K Wong, J. H. Lim, X. Jia, F. Yin, H. Li, W. Xiong, T.Y. Wong, "Optic Cup and Disc extraction from Retinal Fundus Images for Determination of Cup- to- Disc Ratio, " in proceedings of 2008, IEEE Engineering pp 1828-1832.

[10] Zhuo Zhang ,Jiang Liu , Cherian, N.S. ,Ying Sun, Joo Hwee Lim , Wing Kee Wong, Ngan Meng Tan ,Shijian Lu , Huiqi Li , Tien Ying Wong, "Convex hull based neuro-retinal optic cup ellipse optimization in glaucoma diagnosis", Engineering in Medicine and Biology Society, 2009. EMBC2009. Annual International Conference of the IEEE, Inst. for Infocomm Res., A*STAR, Singapore, 3-6 Sept. 2009, pp. 1441 - 1444, 2009.

[11] Wong, D.W.K. ; Jiang Liu ; Joo Hwee Lim ; Ngan Meng Tan ; Zhuo Zhang ; Huiqi Li ; Shijian Lu ; Tien Yin Wong, 'Method of detecting kink-bearing vessels in a retinal fundus image(CDR)', The 5th IEEE Conference on Industrial Electronics and Applications (ICIEA), pp. 1690 - 1694, June 2010

[12] Joshi, G.D., Sivaswamy, J., Karan, K., Prashanth, R., Krishnadas, S.R., "Vessel Bend-Based Cup Segmentation in Retinal Images", 20th International Conference on Pattern Recognition (ICPR), 2010, CVIT, IIIT Hyderabad, Hyderabad, India, 23-26 Aug. 2010, pp. 2536 - 2539, 2010.

[13] Shijian Lu and Joo Hwee Lim, "Automatic optic disc detection through background estimation" Proceedings of 2010 IEEE 17th International Conference on Image Processing, Hong Kong, September 26-29, 2010.

[14] Gopal Datt Joshi, Jayanthi Sivaswamy, Kundan Karan, S. R. Krishnadas, "Optic Disk And Cup Boundary Detection Using Regional Information" Proceedings of the 2010 IEEE international conference on Biomedical imaging: from nano to Macro,2010.

[15] Angel Suero, Diego Marin, Manuel E. Gegundez-Arias, and Jose M. Bravo, "Locating the Optic Disc in Retinal Images Using Morphological Techniques", IWBBIO 2013 Proceedings, Granada, 18-20 March, 2013, pp.593-600, 2013.

[16] N. M. Salem and A. K. Nandi, "Novel and adaptive contribution of the red channel in pre-processing of colour fundus images," in Journal of the Franklin Institute, 2007, p. 243256, 2007.

[17] Rafael C Gonzalez, Richard E Woods, Steven L Eddins, Digital Image Processing, Prentice Hall Publications, 2008.

[18] Rafael C Gonzalez, Richard E Woods, Steven L Eddins., Digital Image Processing Using Matlab, Prentice Hall Publications,2008.

[19] HeikoTimm, Christian Borgelt, and Rudolf KruseFuzzy, "Cluster Analysis with Cluster Repulsion", CiteSeerx.

[20] Yinghua Lu, Tinghuai Ma, Changhong Yin, Xiaoyu Xie, Wei Tian,ShuiMing Zhong, "Implementation of the Fuzzy C-Means Clustering Algorithm in Meteorological Data", International Journal of Database Theory and Application, Vol.6, No.6, pp.1-18, 2013

[21] Thresiamma Devasia, Poulose Jacob, Tessamma Thomas, "Automatic Optic Disc Boundary Extraction from Color Fundus Images", International Journal of Advanced Computer Science and Applications (IJACSA), Vol. 5, No. 7, 2014,pp.17-24

\section{AUTHORS}

Thresiamma Devasia was graduated with Bachelor of Mathematics (BSc.Maths) from Mahatma University, Kerala, India in 1991, and finished her Master of Computer Applications (MCA) and M.Phil Computer Science from Alagappa University Tamilnadu, India in 1995 and 2010, respectively. Currently, she is the Head and Associate professor, Department of Computer Science at Assumption College Changanacherry, Kerala, India and working toward her Ph.D. at Cochin University of Science And Technology on glaucoma detection using image processing. She completed UGC sponsored minor research project based on image processing. She was a member of IEEE. Her interest areas include image processing and medical imaging.

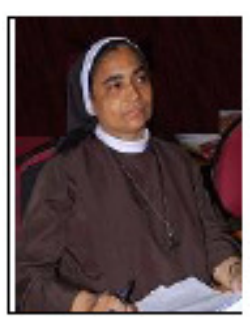


Signal \& Image Processing : An International Journal (SIPIJ) Vol.6, No.3, June 2015

Dr. K.Poulose Jacob, Professor of Computer Science at Cochin University of Science and Technology since 1994, is currently Pro Vice Chancellor of Cochin University of Science \& Technology. He has presented research papers in several International Conferences in Europe, USA, UK, Australia and other countries. He has served as a Member of the Standing Committee of the UGC on Computer Education \& Development. He is the Zonal Coordinator of the DOEACC Society under the Ministry of Information Technology, Government of India. He serves as a member of the AICTE expert panel for accreditation and approval. He has been a member of

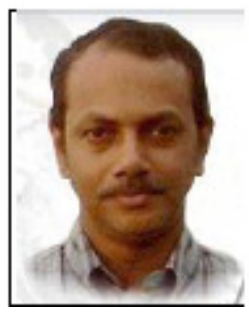
several academic bodies of different Universities and Institutes. He is on the editorial board of two international journals in Computer Science. Dr. K.Poulose Jacob is a Professional member of the ACM (Association for Computing Machinery) and a Life Member of the Computer Society of India.

Dr.Tessamma Thomas received her M.Tech. and Ph.D from Cochin University of Science and Technology, Cochin-22, India. At present she is working as Professor in the Department of Electronics, Cochin University of Science and Technology. She has to her credit more than 100 research papers, in various research fields, published in International and National journals and conferences. Her areas of interest include digital signal / image processing, bio medical image processing, super resolution, content based image retrieval, genomic signal processing, etc.

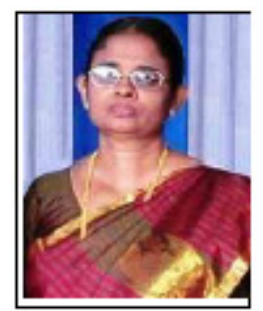

